\title{
A MODEL-BASED SCHEME FOR ANTICONTROL OF SOME DISCRETE-TIME CHAOTIC SYSTEMS
}

\author{
ÖMER MORGÜL \\ Bilkent University, \\ Department of Electrical and Electronics Engineering, \\ 06800, Bilkent, Ankara, Turkey \\ morgul@ee.bilkent.edu.tr
}

Received October 16, 2002; Revised August 26, 2003

\begin{abstract}
We consider a model-based approach for the anticontrol of some discrete-time systems. We first assume the existence of a chaotic model in an appropriate form. Then by using an appropriate control input we try to match the controlled system with the chaotic system model. We also give a procedure to generate the model chaotic systems in arbitrary dimensions. We show that with this approach, controllable systems can always be chaotified. Moreover, if the system to be controlled is stable, control input can be chosen arbitrarily small.
\end{abstract}

Keywords: Chaotic systems; chaos control; chaotification; anticontrol; chaos synchronization.

\section{Introduction}

The analysis and control of chaotic behavior in dynamical systems has been investigated by many researchers in various disciplines in recent years. Among the vast amount of works already published in the literature, the interested reader may consult e.g. various survey papers such as [Fradkov \& Evans, 2002; Boccaletti et al., 2000; Gadre \& Varma, 1997; Chen \& Moiola, 1994], to research monographs such as [Kapitaniak, 2000; Chen \& Dong, 1998; Fradkov \& Pogromsky, 1998], and to a bibliography [Chen, 1996].

While in majority of works in the area of chaos control, the main aim is the suppression of chaotic behavior, see e.g. [Fradkov \& Evans, 2002; Chen \& Dong, 1998], the opposite approach, i.e. to retain the chaotic behavior, or even to force a regular behavior into a chaotic one, has also received considerable interest. This problem is known as "anticontrol" [Schiff et al., 1994], or "chaotification" [Wang \& Chen, 2000a], and has a great potential for applications in diverse fields, see e.g. [Brandt \& Chen, 1997; Ditto, 1996; Goldberger, 1994; Yang et al., 1995]. Various feedback schemes, mostly for discrete-time systems are available in the literature for the anticontrol of such systems, see e.g. [Chen \& Lai, 1996, 1998; Wang \& Chen, 2000b].

In this work, we will consider a model-based approach for the anticontrol of some discrete-time systems. We first assume the existence of a chaotic model in an appropriate form. Then by using an appropriate control input we try to match the controlled system with the chaotic system model. We prove that (i) any controllable linear time-invariant system can be chaotified with an appropriate input, (ii) this approach could be generalized to a class of nonlinear systems, (iii) if in addition the system to be controlled is stable, then the control input can be chosen arbitrarily small. We also address the question of the existence of model chaotic systems. We propose a simple procedure to generate such chaotic models in arbitrary dimension. Then we consider the computability of the required feedback law by using only the available signals. For this aim, estimates of the controlled system states could be used and such estimates could be obtained by using an 
appropriate synchronization scheme. As an example, we propose an observer-based synchronization scheme. We also comment on the robustness of the proposed scheme. We note that this approach could also be applied to the anticontrol of continuous-time systems, see [Morgül, 2003].

This paper is organized as follows. In the second section, we define the problems considered in this paper and present some developments which will be used in the sequel. In the third section, we propose an anticontrol scheme for linear systems, and then generalize it to a class of nonlinear systems. In the fourth section, we present a method to compute the required control input when the available output is not sufficient. For this aim, we propose an observer-based synchronization scheme to estimate the states of the system to be controlled. In the fifth section, we propose a simple way to generate the model chaotic systems for arbitrary dimension. Then we consider the problem of chaotification by arbitrary small control input. In the following section we present some simulation results. Finally we give some concluding remarks.

\section{Problem Statement}

We will first consider the linear systems. Consider the system given below:

$$
x(k+1)=A x(k)+B u(k), \quad y(k)=C x(k),
$$

where $x \in \mathbf{R}^{n}, A \in \mathbf{R}^{n \times n}$ is a constant matrix, $B, C^{T} \in \mathbf{R}^{n}$ are constant vectors, here superscript $T$ denotes transpose, $u$ is the (scalar) control input and $y$ is the (scalar) output, which is assumed to be measurable, and $k=0,1,2, \ldots$ is an integer. For this system, we pose the following problems.

Problem 1. Find a feedback law $u(k)=g(x(k))$, where $g: \mathbf{R}^{n} \rightarrow \mathbf{R}$ is an appropriate function, such that the resulting closed-loop system exhibits chaotic behavior.

Problem 2. Assume that the feedback law $u(k)=$ $g(x(k))$, which solves Problem 1, cannot be computed by using the output $y(k)$ alone. Find an approximate control law $u(k)=\hat{u}(k)$, which can be computed by using the output, such that $\| \hat{u}(k)-$ $g(x(k)) \| \rightarrow 0$ as $k \rightarrow \infty$; here $x(k)$ is the solution of (1), and $\|\cdot\|$ denotes any norm in $\mathbf{R}^{n}$.

In the next section we will provide a solution to Problem 1. In Sec. 4 we will propose a synchronization based solution for Problem 2. In this approach, we will use an observer-based scheme to estimate $x(k)$ and the output $y(k)$ will be used as a synchronization signal. These estimates will then be used to approximate the control law $u(k)=g(x(k))$.

For simplicity, we will first transform the system given by (1) into an appropriate canonical form. Let us define the following matrix:

$$
Q_{c}=\left(\begin{array}{lll}
A^{n-1} B & A^{n-2} B \cdots & A B B
\end{array}\right) .
$$

It is well-known that the system given by (1) is controllable (i.e. any state $x_{0} \in \mathbf{R}^{n}$ can be steered to any state $x_{1} \in \mathbf{R}^{n}$ with an appropriate control input $u$ ) if and only if $\operatorname{rank}\left(Q_{c}\right)=n$, see e.g. [Kailath, 1980]. We will assume that this condition holds, hence $Q_{c}$ is assumed to be invertible.

Let $p(\lambda)$ be the characteristic polynomial of $A$ given by (1), which is given as follows:

$$
\begin{aligned}
p(\lambda) & =\operatorname{det}(\lambda I-A) \\
& =\lambda^{n}+\alpha_{1} \lambda^{n-1}+\cdots+\alpha_{n-1} \lambda+\alpha_{n} .
\end{aligned}
$$

Now, let us define the vectors $u_{1}=\left(\begin{array}{lll}1 & \alpha_{1} \ldots \alpha_{n-1}\end{array}\right)^{T}$, $u_{2}=\left(\begin{array}{llll}0 & 1 & \alpha_{1} \ldots \alpha_{n-2}\end{array}\right)^{T}, \ldots, u_{n}=\left(\begin{array}{llll}0 & 0 & \ldots\end{array}\right)^{T}$, and define the matrices $U=\left(u_{1} u_{2} \ldots u_{n}\right), R=$ $\left(Q_{c} U\right)^{-1}$. By using the coordinate transformation $z=R x$, (1) can be transformed into the following form:

$$
z(k+1)=\hat{A} z+\hat{B} u, \quad y=\hat{C} z,
$$

where $z=\left(\begin{array}{llll}z_{1} & z_{2} & \ldots & z_{n}\end{array}\right)^{T}, \hat{A}=R A R^{-1}, \hat{B}=$ $R B, \hat{C}=C R^{-1}$. After straightforward calculations and by using Cayley-Hamilton theorem (i.e. $p(A)=$ 0 , where $p(\cdot)$ is given by $(3))$, it can be shown that $\hat{A}$ and $\hat{B}$ have the following form:

$$
\begin{aligned}
\hat{A} & =\left(\begin{array}{ccccc}
0 & 1 & 0 & \ldots & 0 \\
0 & 0 & 1 & \ldots & 0 \\
& & \vdots & & \\
0 & 0 & 0 & \ldots & 1 \\
-\alpha_{n} & -\alpha_{n-1} & -\alpha_{n-2} & \ldots & -\alpha_{1}
\end{array}\right), \\
\hat{B} & =\left(\begin{array}{c}
0 \\
0 \\
\vdots \\
0 \\
1
\end{array}\right) .
\end{aligned}
$$

We note that the form given above is known as controllable canonical form in control theory, see e.g. [Kailath, 1980]. 


\section{An Anti-Control Scheme}

Let us assume that our model chaotic system is given as follows:

$$
\begin{aligned}
w_{1}(k+1) & =w_{2}(k) \\
w_{2}(k+1) & =w_{3}(k) \\
& \vdots \\
w_{n-1}(k+1) & =w_{n}(k) \\
w_{n}(k+1) & =f\left(w_{1}(k), w_{2}(k), \ldots, w_{n}(k)\right)
\end{aligned}
$$

where $f: \mathbf{R}^{n} \rightarrow \mathbf{R}$ is an appropriate function. For $n=1$, the system given by (6) reduces to $w(k+1)=f(w(k))$, and there are many onedimensional chaotic systems which have this form, e.g. logistic equation. For $n=2$, the well-known Hénon system can be easily transformed into this form. In Sec. 5, we will propose a simple scheme to generate chaotic systems of this form for arbitrary dimension $n>1$.

Note that (6) could be rewritten as

$$
w(k+1)=\hat{A} w(k)+\hat{B} h(w(k)),
$$

where $w=\left(\begin{array}{llll}w_{1} & w_{2} & \ldots & w_{n}\end{array}\right)^{T}$, and

$$
\begin{aligned}
h(w(k))= & f(w(k))+\alpha_{1} w_{n}(k)+\alpha_{2} w_{n-1}(k) \\
& +\cdots+\alpha_{n} w_{1}(k) .
\end{aligned}
$$

Here, $\alpha_{i}$ are arbitrary constants.

Our anticontrol scheme is based on matching the system given in (4) with the model chaotic system given in (6) by using an appropriate control input $u(k)$. Hence, to achieve this goal, we may choose $u(k)$ as:

$$
\begin{aligned}
u(k)= & h(z(k)) \\
= & f(z(k))+\alpha_{1} z_{n}(k)+\alpha_{2} z_{n-1}(k) \\
& +\cdots+\alpha_{n} z_{1}(k) .
\end{aligned}
$$

Obviously, by using (9) we can transform (4) into the chaotic system given in (6).

The anticontrol scheme given above can also be applied to a class of nonlinear systems as well. Let us assume that the system to be controlled is given as:

$$
\begin{aligned}
x(k+1) & =A(x(k))+B(x(k)) u(k), \\
y(k) & =C(x(k)),
\end{aligned}
$$

where $A, B: \mathbf{R}^{n} \rightarrow \mathbf{R}^{n}$ and $C: \mathbf{R}^{n} \rightarrow \mathbf{R}$ are appropriate functions, $u$ and $y$ are control input and measurement outputs, respectively, which are scalars. Let us assume that there exists a coordinate change
$z=T(x)$, where $T: \mathbf{R}^{n} \rightarrow \mathbf{R}^{n}$ is an appropriate function, which transforms (10) into the following form:

$$
\begin{aligned}
z(k+1) & =\hat{A} z(k)+\hat{B}(\gamma(z(k))+\beta(z(k)) u(k)), \\
y(k) & =\hat{C}(z(k)),
\end{aligned}
$$

where $\hat{A}, \hat{B}$ are as given in $(5), \alpha_{i}, i=1, \ldots, n$ are appropriate constants, and $\gamma, \beta, \hat{C}: \mathbf{R}^{n} \rightarrow \mathbf{R}$ are appropriate functions. Note that the terms multiplying $\alpha_{i}$ in (11) could be included in $\gamma(\cdot)$. The transformation given above is related to the concept of feedback linearization, and a set of conditions guaranteeing the existence of such a transformation is known, see e.g. [Khalil, 2002].

An appropriate control input $u(k)$ to obtain a model match between (11) and (6) is given as follows:

$$
u(k)=\frac{h(z(k))-\gamma(z(k))}{\beta(z(k))},
$$

where $h(\cdot)$ is given by (8). Obviously, we require $\beta(z(k)) \neq 0$ along the solutions of (11). This requirement is natural, since otherwise the control input $u(k)$ has no effect on the system dynamics, see (11).

The results presented in this section can be summarized as follows

(i) Any controllable linear (single input) system can be chaotified with an appropriate control law.

(ii) Any nonlinear (single input) system which could be transformed into the form (11) can be chaotified with an appropriate control law provided that $\beta(z(k)) \neq 0$.

\section{Synchronization-based Implementation}

For the computation of the control laws given in (9) or $(12)$, in general the state vector $z(k)$ should be available through measurements. However, in most cases, the available output signal $y(k)$ is not sufficient alone to compute the required control signal. In such cases, an appropriate approach would be to obtain an approximation $\hat{z}(k)$ of $z(k)$, and use this estimate to approximate the required control signal.

Since the synchronization schemes may provide good estimates of the receiver states, which is $z(k)$ in our case, a natural approach to solve Problem 2 given in Sec. 2 is to use a synchronization scheme for 
the system given in (4). With this aim, any synchronization scheme which uses the output $y(k)$ of (4) as a synchronization signal and provides estimates $\hat{z}(k)$ of $z(k)$ could be used. There are many such schemes proposed in the literature, see e.g. [Ushio, 1999]. For illustrative purposes, we will consider the following observer-based synchronization scheme:

$$
\begin{aligned}
\hat{z}(k+1) & =\hat{A} \hat{z}(k)+\hat{B} u(k)+K(y(k)-\hat{y}(k)), \\
\hat{y}(k) & =\hat{C} \hat{z}(k),
\end{aligned}
$$

where $K \in \mathbf{R}^{n}$ is a gain vector to be determined. Let the synchronization error be defined as $e(k)=$ $z(k)-\hat{z}(k)$. By using (4) and (13) we obtain:

$$
e(k+1)=(\hat{A}-K \hat{C}) e(k) .
$$

Therefore $e(k) \rightarrow 0$ as $k \rightarrow \infty$ if and only if the matrix $A_{c}=\hat{A}-K \hat{C}$ is Schur stable (i.e. any eigenvalue $\lambda$ of $A_{c}$ satisfies $\left.|\lambda|<1\right)$. Moreover, in this case the decay is exponential, i.e. the following holds for some $M>0$ and $0<\rho<1$ :

$$
\|e(k)\| \leq M \rho^{k}\|e(0)\| .
$$

It is known that there exists such a gain vector $K$ which makes $A_{c}$ Schur stable if the system given in (1), or equivalently the system given in (4), is observable, see e.g. [Kailath, 1980]. It is also known that the latter condition is satisfied if and only if the observability matrix $Q_{o}$ given below has full rank:

$$
Q_{o}=\left(\begin{array}{c}
C \\
C A \\
\vdots \\
C A^{n-1}
\end{array}\right) .
$$

see e.g. [Kailath, 1980]. Moreover, in this case the decay rate $\rho$ given in (15) can be assigned arbitrarily. Note that this condition is sufficient in many observer-based synchronization schemes for continuous-time systems, see [Morgül \& Solak, 1996, 1997; Morgül, 1999].

As explained above, a natural approximation of the control law given in (9) is to use $\hat{z}(k)$ instead of $z(k)$, i.e. to use $u=h(\hat{z}(k))$. To see the effect of this approximation, let us assume that $h: \mathbf{R}^{n} \rightarrow \mathbf{R}$ given in (9) is a Lipschitz function, i.e. the following holds for some $c>0$ :

$$
\|h(z)-h(\hat{z})\| \leq c\|z-\hat{z}\| .
$$

Let us assume that the feedback law $u=h(\hat{z}(k))$ is used in (4). Since $h(\hat{z}(k))=h(\hat{z}(k))-h(z(k))+$ $h(z(k))$, from (4) we obtain the following:

$$
z(k+1)=\hat{A} z(k)+\hat{B} h(z(k))+e_{c}(k),
$$

where the error term $e_{c}(k)=\hat{B}(h(\hat{z}(k))-h(z(k)))$ satisfies

$$
\left\|e_{c}(k)\right\| \leq c M \rho^{k}\|e(0)\|,
$$

here we used (15) and (17). Since the perturbation term in (18) is exponentially decaying, it is natural to expect that the qualitative behavior of (18) and (7) be similar, provided that the chaotic behavior is structurally stable. If the chaotic attractor of (7) is globally attractive, then the solutions of (18) will eventually converge to this attractor since $e_{c}(k) \rightarrow$ 0 as $k \rightarrow \infty$. On the other hand, if the chaotic attractor of (7) is only locally attractive, then only local convergence maybe valid. To elaborate further, let us assume that the chaotic attractor of (7) is only locally attractive and is structurally stable, in the sense that for some $\varepsilon>0$, the behaviors of (7) and (18) are qualitatively similar provided that $\left\|e_{c}(k)\right\| \leq \varepsilon$, see e.g. [Fradkov \& Pogromsky, 1998]. It easily follows from (19) that this condition holds for $\|e(0)\| \leq \varepsilon / c M$. Therefore, if the initial error is sufficiently small then the solutions of (18) will be chaotic provided that the chaotic attractor of (7) is locally attractive and structurally stable in the sense given above. On the other hand, if $\|e(0)\| \leq R$ for some $R>0$, it follows from (19) that $\left\|e_{c}(k)\right\| \leq \varepsilon$ for $k>N=(\ln \varepsilon-\ln c M R) / \ln \rho$. Hence we could use a switching law to generate $u$ as follows:

$$
u(k)= \begin{cases}0 & k<N \\ h(\hat{z}(k)) & k \geq N\end{cases}
$$

If the system to be controlled is nonlinear and is given by (11), then the method given above could be used provided that a synchronization scheme for (11) is available. Since our main aim is to provide an anticontrol scheme, we do not elaborate on this point. For such a synchronization scheme, see e.g. [Ushio, 1999]. We also note that the basic idea presented above is similar to the observer-based control of some chaotic systems presented in [Solak et al., 2001].

Remark 1. The requirement that various functions be Lipschitz may seem to be restrictive. Note that any differentiable function is locally Lipschitz in any bounded domain. Hence, if the solutions remain in a bounded set, which is the case for chaotic systems, then this requirement is satisfied provided that the corresponding functions are differentiable. 
Remark 2. Let us consider the effect of parameter mismatch in the anticontrol scheme proposed above. Such a case may arise if the parameters of the controlled system are not known exactly. In such cases, the effect of such discrepancies may be included in the system equations by adding an extra perturbation term $e_{p}(k)$, similar to $e_{c}(k)$ in $(18)$. Note that $\left\|e_{p}(k)\right\|$ will be proportional to the magnitude of parameter mismatch. Therefore, this perturbation term will be small provided that the parameter mismatch magnitude is small, and hence will not affect the proposed anticontrol scheme. Another source of such an error term may be the possible parameter mismatch and noise which could be present in the synchronization scheme. Such effects may also be included in the system dynamics by adding yet another perturbation term. This extra term will also be small provided that the parameter mismatch magnitudes and noise magnitudes are small and the synchronization scheme is robust with respect to noise and parameter mismatch.

\section{Model Chaotic Systems}

In the sequel we will present a simple scheme to generate chaotic systems of the form given by (6) in arbitrary dimension $n$. Note that for $n=1$, the required form reduces to $w(k+1)=f(w(k))$, and there are many one-dimensional chaotic systems in this form. Now assume that there exists a chaotic system of the form given by (6) for $n \geq 1$. We will present a simple scheme to generate a chaotic system of the same form for dimension $n+1$. Consider the following system:

$$
\begin{aligned}
w_{1}(k+1) & =w_{2}(k) \\
w_{2}(k+1) & =w_{3}(k) \\
& \vdots \\
w_{n-1}(k+1) & =w_{n}(k) \\
w_{n}(k+1) & =f\left(w_{1}(k), w_{2}(k), \ldots, w_{n}(k)\right)+z(k) \\
z(k+1) & =\rho z(k)
\end{aligned}
$$

where $|\rho|<1$ is an arbitrary real number. Obviously, $z(k)=\rho^{k} z(0) \rightarrow 0$ as $k \rightarrow \infty$, hence the first $n$ equations of (21) and (6) are asymptotically the same. Therefore if (6) has a globally attractive chaotic attractor, so does (21). On the other hand, if (6) has only locally attractive chaotic attractor, which is structurally stable in the sense given in Sec. 4, then so does (21) provided that $|z(0)|$ is sufficiently small.

To transform (21) into the form (6), let us define the variable $w_{n+1}$ as follows:

$$
w_{n+1}(k)=f\left(w_{1}(k), w_{2}(k), \ldots, w_{n}(k)\right)+z(k) .
$$

Hence, from (21) we have $w_{n}(k+1)=w_{n+1}(k)$. By using (21) and (22) we obtain the following:

$$
\begin{aligned}
& w_{n+1}(k+1) \\
&= f\left(w_{1}(k+1), w_{2}(k+1), \ldots, w_{n}(k+1)\right) \\
&+z(k+1) \\
&= f\left(w_{2}(k), w_{3}(k), \ldots, w_{n+1}(k)\right)+\rho z(k) \\
&= f\left(w_{2}(k), w_{3}(k), \ldots, w_{n+1}(k)\right)+\rho w_{n}(k+1) \\
&-\rho f\left(w_{1}(k), w_{2}(k), \ldots, w_{n}(k)\right) \\
&= f\left(w_{2}(k), w_{3}(k), \ldots, w_{n+1}(k)\right)+\rho w_{n+1}(k) \\
&-\rho f\left(w_{1}(k), w_{2}(k), \ldots, w_{n}(k)\right) .
\end{aligned}
$$

Hence, (21) can be rewritten as follows:

$$
\begin{aligned}
w_{1}(k+1) & =w_{2}(k) \\
w_{2}(k+1) & =w_{3}(k) \\
& \vdots \\
w_{n}(k+1) & =w_{n+1}(k) \\
w_{n+1}(k+1) & =F\left(w_{1}(k), w_{2}(k), \ldots, w_{n+1}(k)\right)
\end{aligned}
$$

where $F$ is given as:

$$
\begin{aligned}
F(w(k))= & f\left(w_{2}(k), w_{3}(k), \ldots, w_{n+1}(k)\right) \\
& +\rho w_{n+1}(k)-\rho f\left(w_{1}(k),\right. \\
& \left.w_{2}(k), \ldots, w_{n}(k)\right) .
\end{aligned}
$$

As an application, consider the well-known Hénon system given below:

$$
\begin{aligned}
& x(k+1)=1+y(k)-a x^{2}(k) \\
& y(k+1)=b x(k) .
\end{aligned}
$$

Let us define the new variables as $w_{1}=y, w_{2}=b x$. Then, (25) can be transformed into the following form:

$$
\begin{aligned}
& w_{1}(k+1)=w_{2}(k) \\
& w_{2}(k+1)=b+b w_{1}(k)-\frac{a}{b} w_{2}^{2}(k),
\end{aligned}
$$


which has the form given in (6) for $n=2$. By using (6), (24), (25) and (27), we obtain the following chaotic system for $n=3$ :

$$
\begin{aligned}
& w_{1}(k+1)=w_{2}(k) \\
& w_{2}(k+1)=w_{3}(k) \\
& w_{3}(k+1)=F\left(w_{1}(k), w_{2}(k), w_{3}(k)\right),
\end{aligned}
$$

where $F$ is given by (see (25)):

$$
\begin{aligned}
F(w(k))= & b-\rho b-\rho b w_{1}(k)+b w_{2}(k)+\rho w_{3}(k) \\
& +\frac{a \rho}{b} w_{2}^{2}(k)-\frac{a}{b} w_{3}^{2}(k) .
\end{aligned}
$$

Remark 3. Note that the system given in (24) will have a chaotic attractor which is qualitatively similar to the attractor of the generating system given in (6). Hence, from mathematical point of view, the system given in (24) is not anymore interesting than the generating lower dimensional model given by (6). However, our aim in this section is not to generate interesting higher dimensional chaotic systems but to show the existence of chaotic systems of the form given in (6) in arbitrary dimensions. Obviously, for our anticontrol scheme any chaotic system which has this form could be used.

\section{Chaotification by Arbitrary Small Feedback}

In this section we will show that when the system given in (4) is stable in the uncontrolled case (i.e. when $u=0$ ), then chaotification is possible with arbitrary small control input. More precisely, under the stated stability assumption, given any $\varepsilon>0$, one can find a control input $u$ satisfying $|u(k)|<\varepsilon$ such that the resulting closed loop system exhibits chaotic behavior. The idea behind this argument is as follows. First note that the size of the chaotic attractor of (6) can be scaled arbitrarily by using a linear transformation in the form $\hat{w}_{i}=\alpha w_{i}$, $i=1,2, \ldots, n$, where $\alpha>0$ is a scaling constant. Since this transformation is linear, the dynamics of the model chaotic system in $\hat{w}$ coordinates will still have the same form given in (6). Since the system to be controlled is stable, we can first choose $u=0$ till the solutions of (6) enter the domain of attraction of the chaotic attractor of the model chaotic system (in $\hat{w}$ coordinates). After this phase, we can apply the control law given in (9). Note that since the size of the chaotic attractor is reduced, the magnitude of $u$ given in (9) will be small as well. Moreover, given the form of $f$, the coefficients $\alpha_{i}$ and the bound $\varepsilon$, one can determine an appropriate scaling constant $\alpha$ by using (9). We note that different strategies to chaotify a given stable linear system are also available in the literature, see e.g. [Wang \& Chen, 2000a, 2000b].

To elaborate further, consider the model chaotic system given in (6). Assume that this system has a chaotic attractor in a compact region $\mathcal{B} \subset \mathbf{R}^{n}$. Without loss of generality, we assume that $\mathcal{B}$ contains the origin, otherwise we could simply increase this domain to satisfy the stated assumption. Let us define the size index $\gamma_{s}$ for the chaotic attractor in question as:

$$
\gamma_{s}=\max \{\|w\| \mid w \in \mathcal{B}\} .
$$

Since we assume that $\mathcal{B}$ is compact, $\gamma_{s}$ is well defined and is finite. Now let us define the following change of variables:

$$
\hat{w}_{i}=\alpha w_{i}, \quad i=1,2, \ldots, n,
$$

where $\alpha>0$ is a scaling constant. By using this change of variables, the dynamics given in (6) is transformed into the following:

$$
\begin{aligned}
\hat{w}_{1}(k+1) & =\hat{w}_{2}(k) \\
\hat{w}_{2}(k+1) & =\hat{w}_{3}(k) \\
& \vdots \\
\hat{w}_{n-1}(k+1) & =\hat{w}_{n}(k) \\
\hat{w}_{n}(k+1) & =\hat{f}\left(\hat{w}_{1}(k), \hat{w}_{2}(k), \ldots, \hat{w}_{n}(k)\right)
\end{aligned}
$$

where $\hat{f}$ is given by:

$$
\hat{f}\left(\hat{w}_{1}(k), \ldots, \hat{w}_{n}(k)\right)=\alpha f\left(\frac{\hat{w}_{1}(k)}{\alpha}, \ldots, \frac{\hat{w}_{n}(k)}{\alpha}\right) .
$$

Clearly, (32) has the same form as given in (6). Therefore (32) also has a chaotic attractor in a compact region $\hat{\mathcal{B}} \subset \mathbf{R}^{n}$. Following (30) and (31), we obtain the following size index $\hat{\gamma}_{s}$ for $\hat{\mathcal{B}}$ :

$$
\hat{\gamma}_{s}=\max \{\|\hat{w}\| \mid \hat{w} \in \hat{\mathcal{B}}\} \leq \alpha \gamma_{s} .
$$

Now consider the system given in (4) and assume that $\hat{A}$ is stable. Hence if we choose $u=0$, the solutions of (4) will satisfy the following:

$$
\|z(k)\| \leq M \rho^{k}\|z(0)\|,
$$

for some $M>1$ and $0<\rho<1$. Hence, eventually we have

$$
\|z(k)\| \leq \hat{\gamma}_{s}, \quad k \geq N=\frac{\ln \frac{\hat{\gamma}_{s}}{M\|z(0)\|}}{\ln \rho} .
$$


Therefore for $k \geq N$, the size of the solutions of (4) become comparable with that of $\hat{\mathcal{B}}$. Hence, for $k \geq N$, we can use the anticontrol scheme given in Sec. 3 by using the model chaotic system given in $(32)$.

Now let us try to find the bound on the control law given in (9). Note that if we use the model chaotic system as given in (32), the required control law becomes:

$u(k)=\hat{f}(\hat{w}(k))+\alpha_{1} \hat{w}_{n}(k)+\cdots+\alpha_{n} \hat{w}_{1}(k)$.

To find a bound on $u$, we need a bound on $\hat{f}$. Now assume that $f$ given in $(6)$ is Lipschitz in $\mathcal{B}$, i.e. the following holds for some $c>0$ :

$$
\|f(w)-f(\tilde{w})\| \leq c\|w-\tilde{w}\|, w, \tilde{w} \in \mathcal{B} .
$$

This condition may seem restrictive. However, if $f$ is (piecewise) differentiable, this condition is automatically satisfied, in fact we have $c \geq$ $\max \{\|D f(w)\| \mid w \in \mathcal{B}\}$, see e.g. [Morgül \& Solak, $1996,1997]$. It then follows from $(31)$ that $\hat{f}$ given in $(33)$ is also Lipschitz in $\hat{\mathcal{B}}$. Moreover, we have:

$$
\begin{aligned}
\|f(\hat{w})-\hat{f}(0)\| & \leq \alpha\|f(\hat{w} / \alpha)-f(0)\| \\
& \leq \alpha c\|\hat{w} / \alpha\| \leq c\|\hat{w}\|,
\end{aligned}
$$

hence we have

$$
\begin{aligned}
\|\hat{f}(\hat{w})\| & \leq\|\hat{f}(0)\|+c\|\hat{w}\| \\
& \leq \alpha\|f(0)\|+c\|\hat{w}\|,
\end{aligned}
$$

for $\hat{w} \in \hat{\mathcal{B}}$. Now by using (34) and (40) in (37), we obtain:

$$
\begin{aligned}
\|u(k)\| & \leq\|\hat{f}(\hat{w}(k))\|+n \max _{i}\left\{\left|\alpha_{i}\right|\right\} \max _{j}\left\{\left\|\hat{w}_{j}(k)\right\|\right\} \\
& \leq \alpha\|f(0)\|+\alpha c \gamma_{s}+n \alpha \gamma_{s} \max _{i}\left\{\left|\alpha_{i}\right|\right\} \\
& \leq \alpha\left(\|f(0)\|+\gamma_{s}\left(c+n \alpha_{m}\right)\right),
\end{aligned}
$$

where $\alpha_{m}=\max _{i}\left\{\left|\alpha_{i}\right|\right\}$. Note that the terms on the left-hand side of (41) are constant. Hence, given $\varepsilon>0$, we can choose $\alpha>0$ sufficiently small so that we have $\|u(k)\| \leq \varepsilon$. In particular, we could choose $\alpha$ as

$$
\alpha \leq \frac{\varepsilon}{\|f(0)\|+\gamma_{s}\left(c+n \alpha_{m}\right)} .
$$

Clearly this idea could be applied to the nonlinear systems of the form given in (11) as well, provided that $\beta(\cdot)$ is bounded away from zero and that $f(\cdot)$ and $\gamma(\cdot)$ are Lipschitz functions. Note that this approach could also be combined with the synchronization-based approach to compute the required control law as given in Sec. 4 .

\section{Simulation Results}

As an example, we consider the following nonlinear system:

$$
\begin{aligned}
x_{1}(k+1) & =c_{1}-x_{2}^{2}(k)-c_{2} x_{3}(k)+u(k) \\
x_{2}(k+1) & =x_{1}(k) \\
x_{3}(k+1) & =x_{2}(k) \\
y(k) & =x_{2}(k),
\end{aligned}
$$

where $u(k)$ and $y(k)$ represent the input and output of the system. This system is called as generalized third-order Hénon map and is known to exhibit chaotic (even hyperchaotic) solutions for certain parameter values when $u(k)=0$ [Baier \& Klein, 1990].

First, we consider the case $c_{1}=1, c_{2}=0.07$, for which (43) exhibits periodic motion when $u(k)=0$, see Fig. 1(a). This system is in the form given in (10) and could be transformed into the form given in (11). One such coordinate change may be given as follows:

$$
z_{1}=x_{3}, \quad z_{2}=x_{2}, \quad z_{3}=x_{1},
$$

By using (44) in (43) we obtain:

$$
\begin{aligned}
z_{1}(k+1) & =z_{2}(k) \\
z_{2}(k+1) & =z_{3}(k) \\
z_{3}(k+1) & =c_{1}-c_{2} z_{1}(k)-z_{2}^{2}(k)+u(k) \\
y(k) & =z_{2}(k) .
\end{aligned}
$$

Note that (45) is in the form given in (11) with $n=3$ and

$$
\alpha_{1}=\alpha_{2}=0, \alpha_{3}=c_{2}, \gamma(z)=c_{1}-z_{2}^{2}, \beta(z)=1 \text {. }
$$

Let us choose the model chaotic system as given in (28). By using the control law given in (12), we choose $u(k)$ in (45) as:

$$
u(k)=F(z(k))+c_{2} z_{1}(k)-\gamma(z(k)),
$$

where $F(\cdot)$ and $\gamma(\cdot)$ are given in (29) and (46), respectively.

Note that the control law given in (47) is not computable by using the available output $y(k)$, see (45). A simple synchronization scheme which uses $y(k)$ as the synchronization signal may be given as follows:

$$
\begin{aligned}
\hat{z}_{1}(k+1)= & \hat{z}_{2}(k)+k_{1}\left(z_{2}(k)-\hat{z}_{2}(k)\right) \\
\hat{z}_{2}(k+1)= & \hat{z}_{3}(k)+k_{2}\left(z_{2}(k)-\hat{z}_{2}(k)\right) \\
\hat{z}_{3}(k+1)= & c_{1}-c_{2} \hat{z}_{1}(k)-z_{2}^{2}(k)+k_{3}\left(z_{2}(k)\right. \\
& \left.-\hat{z}_{2}(k)\right)+u(k)
\end{aligned}
$$




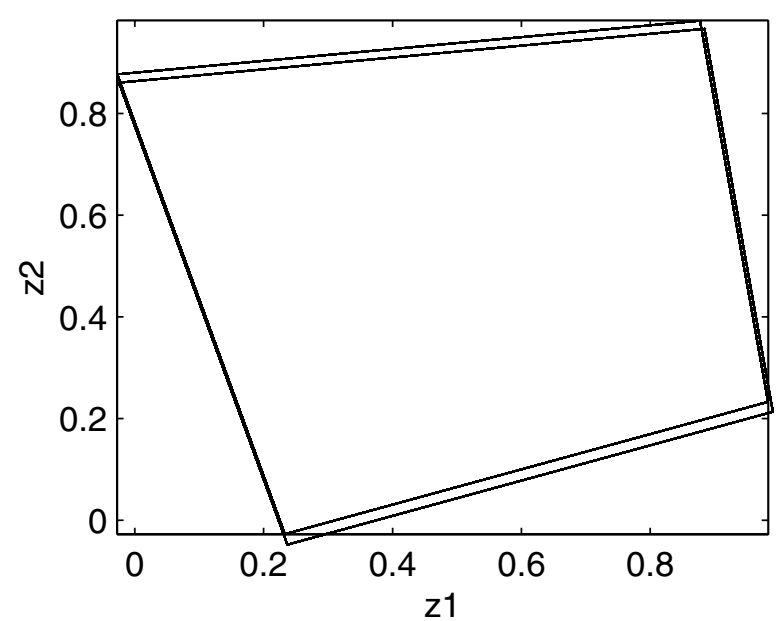

(a)

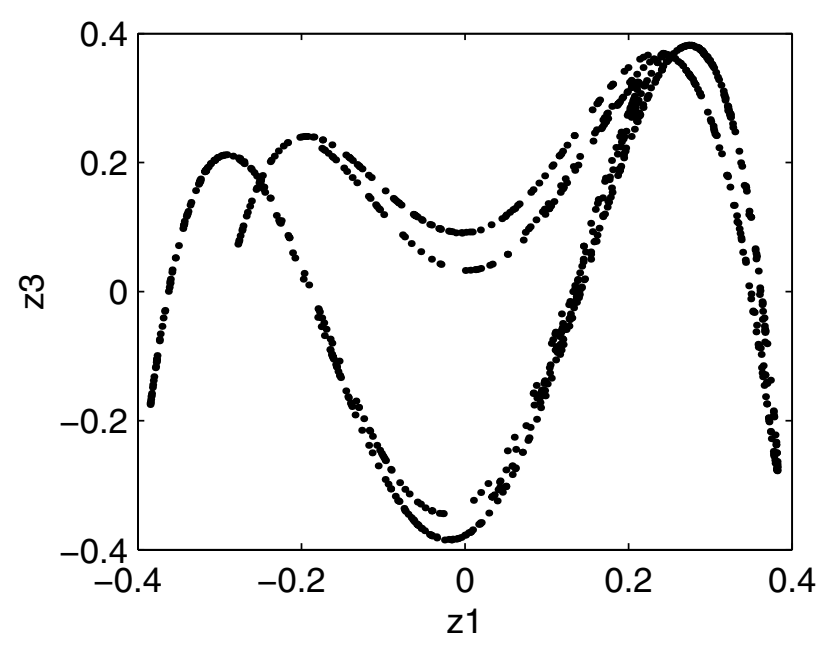

(c)

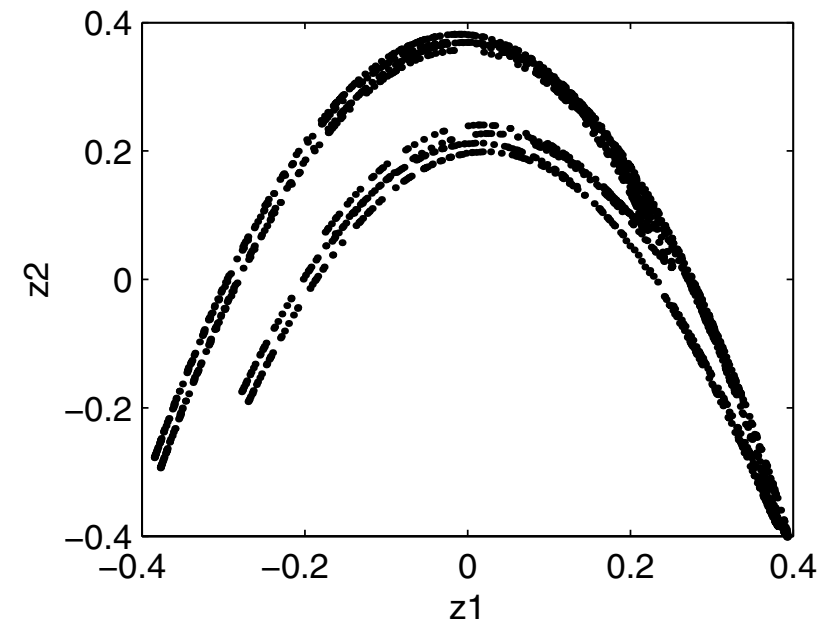

(b)

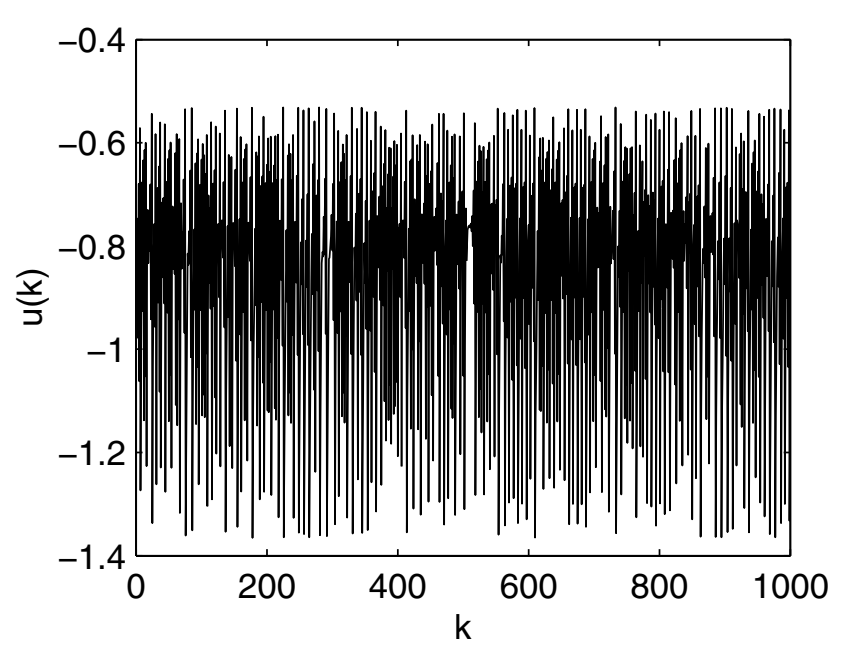

(d)

Fig. 1. Simulation result for generalized Hénon map: ideal case, (a) periodic behavior (without control), (b) $z_{1}$ versus $z_{2}$, (c) $z_{1}$ versus $z_{3},(\mathrm{~d}) u(k)$ versus $k$.

where $K=\left(\begin{array}{lll}k_{1} & k_{2} & k_{3}\end{array}\right)^{T}$ is the gain vector to be determined. A simple calculation shows that the error equation (14) is satisfied. Since the pair $(\hat{C}, \hat{A})$ is observable for $\hat{C}=\left(\begin{array}{lll}0 & 1 & 0\end{array}\right)$, see (45), an appropriate gain vector $K$ which makes $\hat{A}-K \hat{C}$ Schur stable may always be found. For such a selection of $K$, (15) holds, and hence instead of (47), we may use the following control law:

$$
u(k)=F(\hat{z}(k))+c_{2} \hat{z}_{1}(k)-\gamma(z(k)) .
$$

Note that $\gamma(z(k))$ is computable by using $y(k)$, see (45), (46).

In the first set of simulations we considered the ideal case (i.e. the parameter mismatch and noise are not considered). For the system (45) we used $c_{1}=1, c_{2}=0.07$. For the model chaotic system we used $a=1.4, b=0.3$ and $\rho=0.5$. Note that with these parameter choices, the system to be controlled exhibits periodic motion while the model system exhibits chaotic motion. For the observer given in (48) we used $k_{1}=1, k_{2}=0.5, k_{3}=0.4$. For these choices, the eigenvalues of $\hat{A}-K \hat{C}$ are inside the unit circle. We simulated the system given in (45), (48) and (49) with the parameter values stated above. The resulting $z_{1}$ versus $z_{2}$ and $z_{1}$ versus $z_{3}$ graphs are shown in Figs. 1(b) and 1(c), respectively. The required control input given in (49) is also shown in Fig. 1(d).

In the second set of simulations we consider the effect of parameter mismatch and noise on our scheme. To see the effect of noise, we assumed that the synchronization signal $y(k)$ is corrupted 


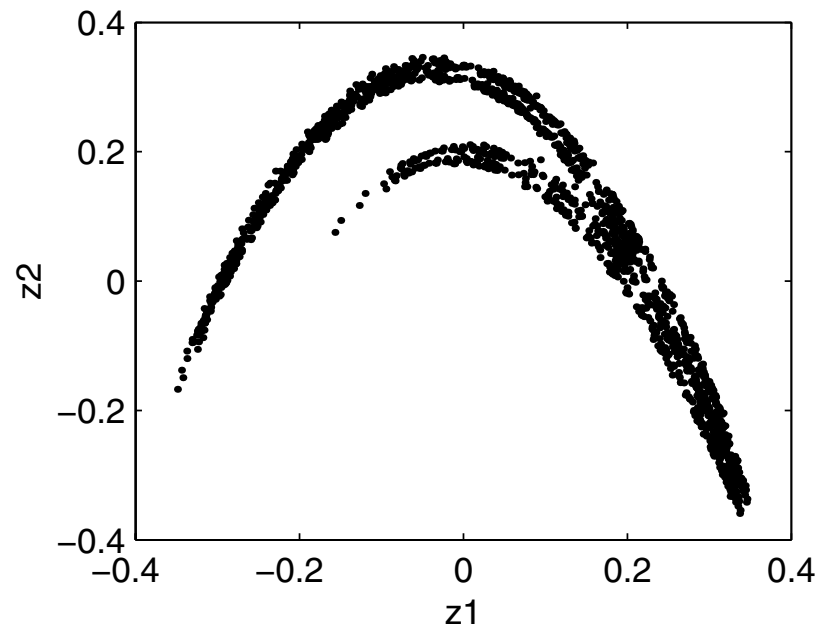

(a)

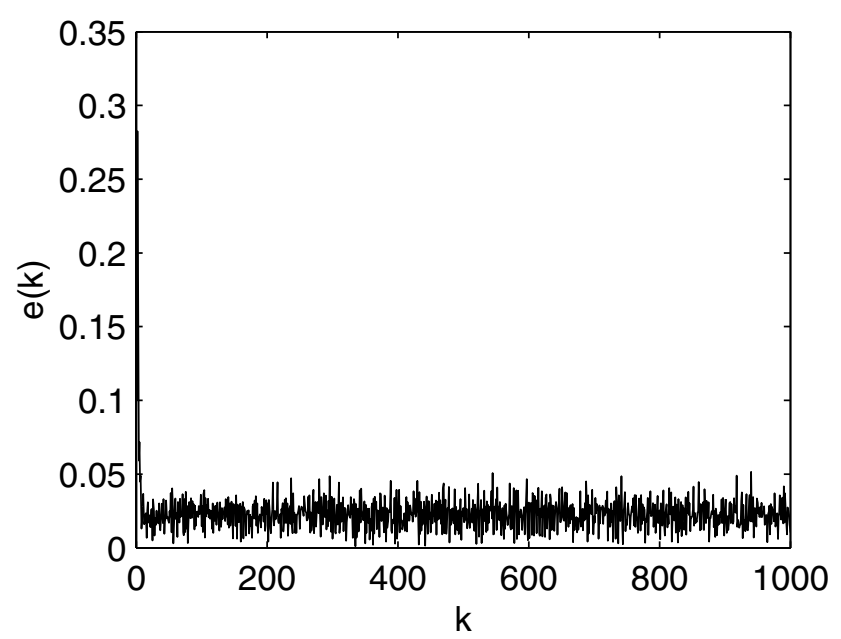

(c)

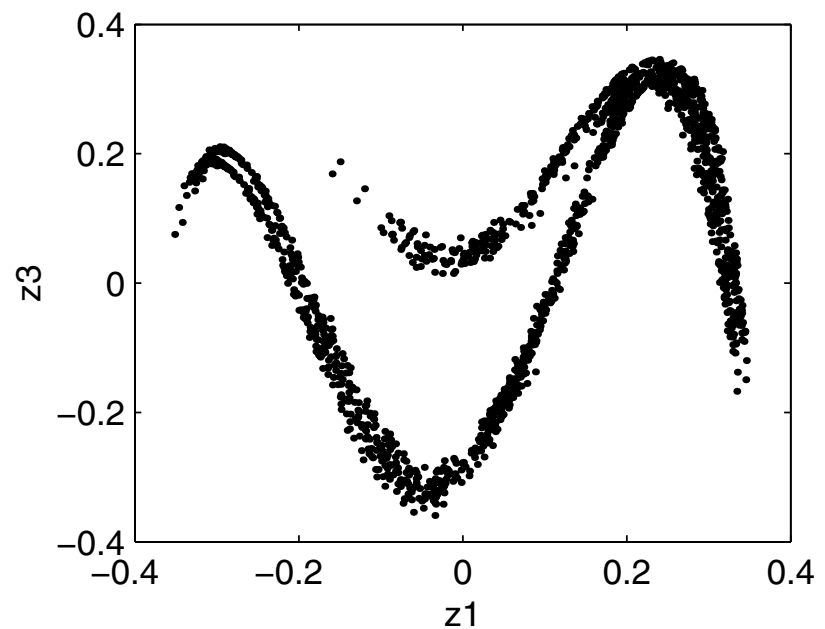

(b)

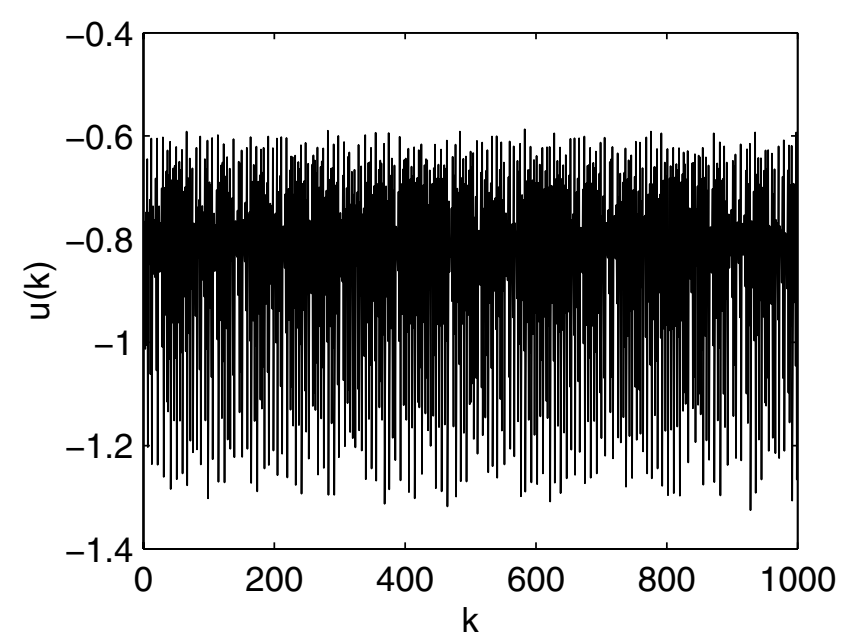

(d)

Fig. 2. Simulation result for generalized Hénon map: nonideal case, (a) $z_{1}$ versus $z_{2}$, (b) $z_{1}$ versus $z_{3}$, (c) $e(k)$ versus $k$, (d) $u(k)$ versus $k$.

by an additive noise. To see this effect, we used $z_{2}(k)+n(k)$ in $(48)$, where $n(k)$ is a random signal uniformly distributed in the interval $\left[\begin{array}{ll}0 & \mathrm{~m}\end{array}\right]$ for some $m>0$. To see the effect of parameter mismatch, we assumed that the model coefficients given in (45) are not known exactly, and instead of the third equation in (48) we used the following

$$
\begin{aligned}
\hat{z}_{3}(k+1)= & (1+\Delta)\left(c_{1}-c_{2} \hat{z}_{1}(k)-\left(z_{2}(k)+n(k)\right)^{2}\right) \\
& +k_{3}\left(z_{2}(k)+n(k)-\hat{z}_{2}(k)\right)+u(k) .
\end{aligned}
$$

This mismatch is also considered in the computation of $u(k)$ as follows:

$$
u(k)=F(\hat{z}(k))+(1+\Delta)\left(c_{2} \hat{z}_{1}(k)-\gamma(z(k))\right),
$$

see (49). We simulated the related system with $m=\Delta=0.02$, and the simulation results are shown in Fig. 2. In addition to $z_{1}$ versus $z_{2}$ and $z_{3}$ graphs shown in Figs. 2(a) and 2(b), respectively, we also show the synchronization error magnitude $e(k)=\|z(k)-\hat{z}(k)\|$ versus $k$, and the required control input $u(k)$ versus $k$ graphs in Figs. 2(c) and 2(d), respectively. As can be seen, the synchronization error is of the same order as the noise and parameter mismatch level, and the proposed scheme is robust with respect to noise and parameter mismatch.

In the last set of simulations, we use the small control input idea given in Sec. 6. For the system to be controlled we consider the nonlinear system given in (45) without the output term $y$. Note that 


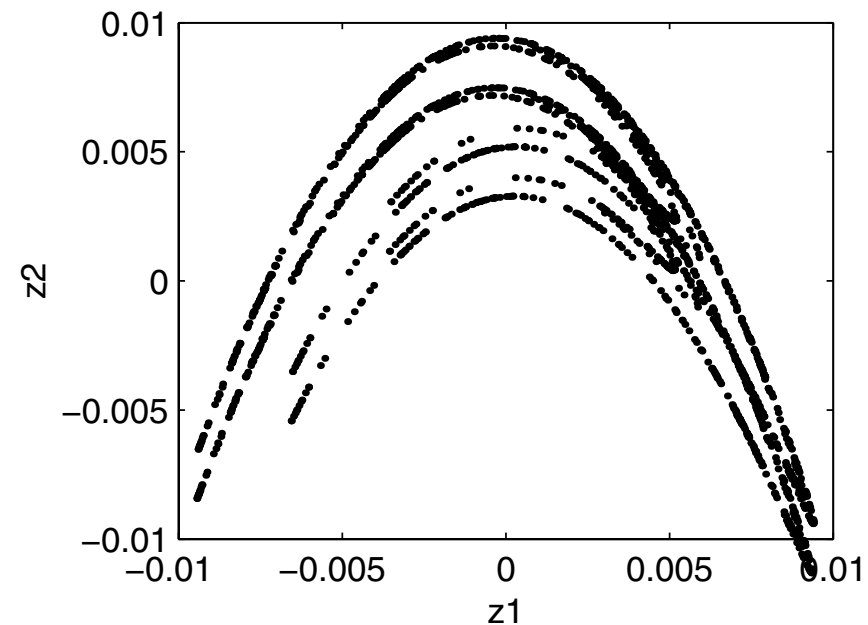

(a)

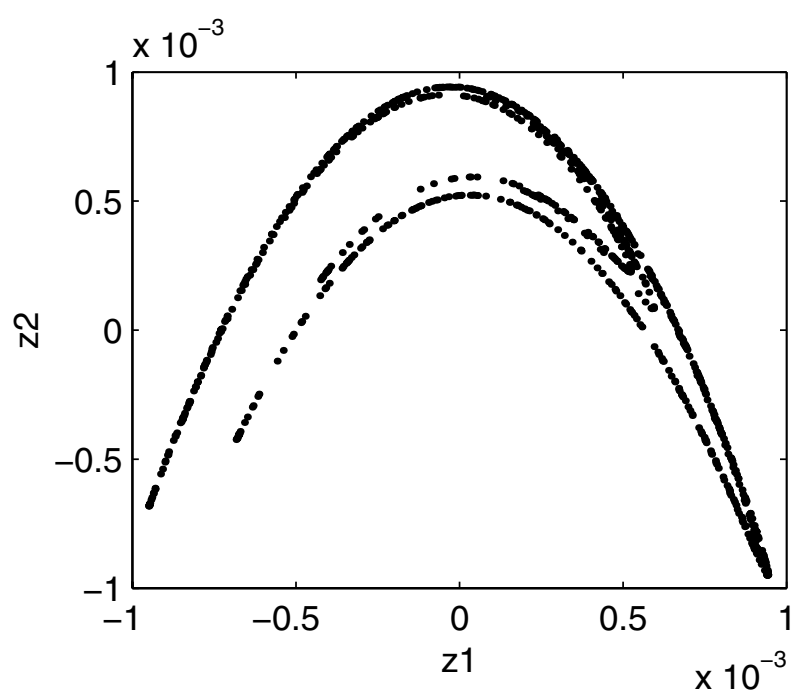

(c)

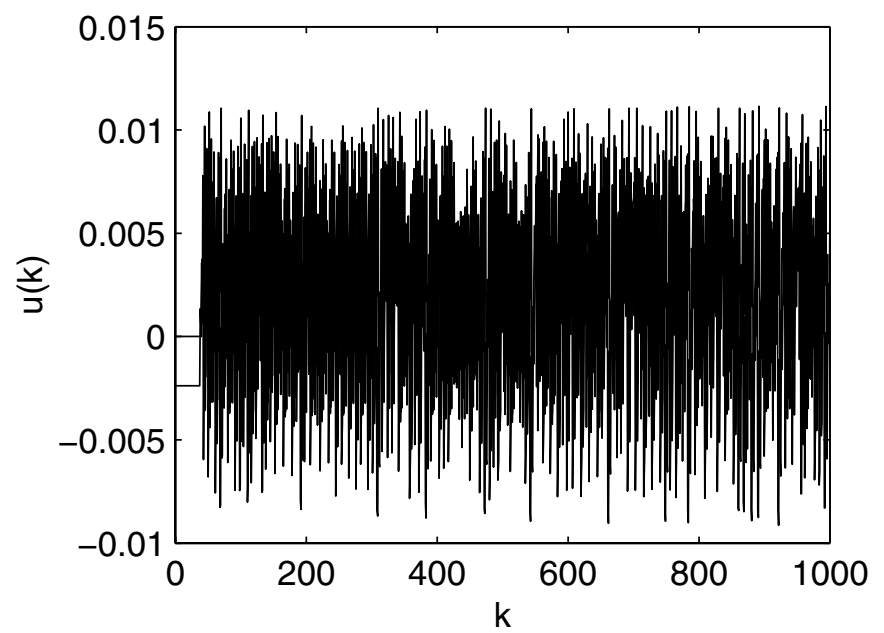

(b)

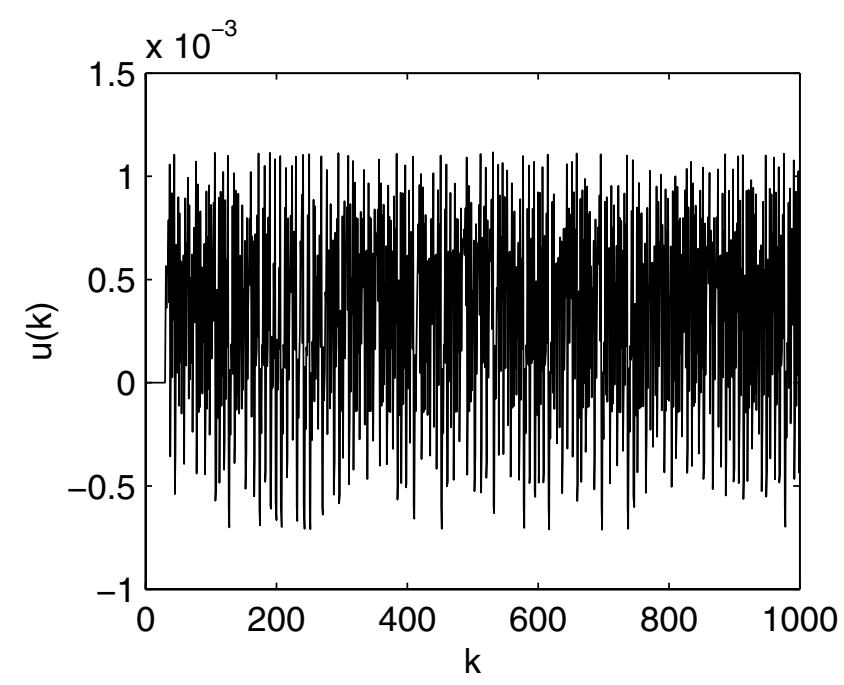

(d)

Fig. 3. Simulation result for small control input, (a) $z_{1}$ versus $z_{2}, \varepsilon=0.1$, (b) $u(k)$ versus $k, \varepsilon=0.1$, (c) $z_{1}$ versus $z_{2}$, $\varepsilon=0.01,(\mathrm{~d}) u(k)$ versus $k, \varepsilon=0.01$.

here we have $\beta(z)=1$, which is bounded away from zero, and both $f(z)$ and $\gamma(z)$ are differentiable, hence Lipschitz in any compact domain. For the parameters $c_{1}=0, c_{2}=0.5$, it can be shown that the origin is asymptotically stable in this system (in the uncontrolled case). The scaled version of the model chaotic system given in (28) can be found as follows:

$$
\begin{aligned}
& \hat{w}_{1}(k+1)=\hat{w}_{2}(k) \\
& \hat{w}_{2}(k+1)=\hat{w}_{3}(k) \\
& \hat{w}_{3}(k+1)=\hat{F}\left(\hat{w}_{1}(k), \hat{w}_{2}(k), \hat{w}_{3}(k)\right),
\end{aligned}
$$

where $\hat{F}$ is given as

$$
\hat{F}\left(\hat{w}_{1}(k), \hat{w}_{2}(k), \hat{w}_{3}(k)\right)=\alpha F\left(\frac{\hat{w}_{1}(k)}{\alpha}, \frac{\hat{w}_{2}(k)}{\alpha}, \frac{\hat{w}_{3}(k)}{\alpha}\right),
$$

and $F$ is given in (29). As before, for the model chaotic system we used $a=1.4, b=0.3$ and $\rho=0.5$. By using simulations, we find that for the case $\alpha=1$, the size of the chaotic attractor as given in (30) is found as $\gamma_{s}=0.6658$. Also, various coefficients in (41) are found as $\|f(0)\|=0.15, c=4.595$. Also, the Lipschitz constant $c_{\gamma}$ of $\gamma(\cdot)$ is estimated as $c_{\gamma}=0.7688$, see (46). By using the ideas given in Sec. 6, we use the following control law:

$$
u(k)= \begin{cases}0 & k<N \\ \hat{F}(z(k))+c_{2} z_{1}(k)-\gamma(z(k)) & k \geq N,\end{cases}
$$

where $\hat{F}$ is given in $(53), \gamma(\cdot)$ is given in (46), and $N$ is given in (36). 
First, we assume that $\varepsilon=0.1$. By using (42), we find that $\alpha \leq 0.0247$, hence we choose $\alpha=0.0247$. Note that since in this example, $\alpha_{1}=\alpha_{2}=0, \alpha_{3} \neq$ 0 , see (46), we may take $n=1$ in (42), see (41). Moreover, $c_{\gamma}$ should be added to $c$, see (54). Initial conditions are chosen as $z_{1}(0)=0.5, z_{2}(0)=$ $z_{3}(0)=0$. By using simulations, it can be found that for $k \geq 20$, the solutions of (45) become comparable with the size of the chaotic attractor of the model system, hence we choose $N=20$ in (54). The results of this simulation are shown in Figs. 3(a) and 3(b). As can be seen, the controlled system exhibits a chaotic behavior (in fact similar to that of a scaled Hénon system), and the control input is bounded by given $\varepsilon$.

Secondly, we assume that $\varepsilon=0.01$. In this case from (42), we find that $\alpha \leq 0.00247$, hence we choose $\alpha=0.00247$. The initial conditions are chosen as given above, and by using simulations we observe that $N=30$ in this case. The results of this simulation are shown in Figs. 3(c) and 3(d). As can be seen, the controlled system exhibits a chaotic behavior and the control input is bounded by given $\varepsilon$.

\section{Conclusion}

In this paper, we considered a model-based approach to the anticontrol of some discrete-time systems. Our aim is to generate a chaotic behavior which is determined by a chaotic model, by means of an appropriate control input. To achieve this task, we assumed the existence of a reference model in an appropriate form which exhibits chaotic behavior. Then we determined an appropriate control input to match the dynamics of the system to be controlled with that of the model chaotic system. We proved that: (i) any controllable linear time-invariant system can be chaotified with an appropriate input, (ii) this approach could be generalized to a class of nonlinear systems, (iii) if in addition the system to be controlled is stable, then the control input can be made arbitrarily small. We proposed a simple procedure to generate such chaotic models in arbitrary dimension. We also considered the computability of the required feedback law by using only the available signals. To estimate the states of the system to be controlled, we proposed a synchronization scheme. Under some mild conditions, exponentially fast synchronization may be achieved, and one can use the estimated states to compute the feedback law. We also commented on the robustness of the proposed scheme. Note that the same approach may also be used in the model reference anticontrol of continuous-time systems, see [Morgül, 2003].

\section{References}

Baier, G. \& Klein, M. [1990] "Maximum hyperchaos in generalized Hénon maps," Phys. Lett. A151, 281-284.

Boccaletti, S., Grebogi, C., Lai, Y. C., Mancini, H. \& Maza, D. [2000] "The control of chaos: Theory and applications," Phys. Rep. 329, 103-197.

Brandt, M. E. \& Chen, G. [1997] "Bifurcation control of two nonlinear models of cardiac activity," IEEE Trans. Circuits Syst.-I 44, 1031-1034.

Chen, G. [1996] "Control and synchronization of chaotic systems, (a bibliography)," ftp.egr.uh.edu/ pub/TeX/chaos.tex loginname: anonymous, password: your e-mail address.

Chen, G. \& Dong, X. [1998] From Chaos to Order: Methodologies, Perspectives and Applications (World Scientific, Singapore).

Chen, G. \& Lai, D. [1996] "Feedback control of Lyapunov exponents for discrete-time dynamical systems," Int. J. Bifurcation and Chaos 6, 1341-1349.

Chen, G. \& Lai, D. [1998] "Feedback anticontrol of discrete chaos," Int. J. Bifurcation and Chaos 8, 1585-1590.

Chen, G. \& Moiola, J. L. [1994] "An overview of bifurcation, chaos, and nonlinear dynamics in nonlinear systems," J. Franklin Inst. B331, 819-858.

Ditto, W. L. [1996] "Applications of chaos in biology and medicine," in Chaos and the Changing Nature of Science and Medicine: An Introduction, ed. Herbert, D. E. (AIP Press, NY), pp. 175-201.

Fradkov, A. L. \& Pogromsky, A. Y. [1998] Introduction to Control of Oscillations and Chaos (World Scientific, Singapore).

Fradkov, A. L. \& Evans, R. J. [2002] "Control of chaos: Survey 1997-2000," in Proc. IFAC 2002 World Congress, July 2002, Barcelona, Spain.

Gadre, S. D. \& Varma, V. S. [1997] "Control of chaos," J. Phys. 48, 259-270.

Goldberger, A. L. [1994] "Applications of chaos to physiology and medicine," in Applied Chaos, eds. Kim, J. H. \& Stringer, J. (Academic Press, NY), pp. 321-331.

Kailath, T. [1980] Linear Systems (Prentice-Hall, NJ).

Kapitaniak, T. [2000] Chaos for Engineers, Theory, Applications and Control (Springer-Verlag, Berlin).

Khalil, H. K. [2002] Nonlinear Systems, 3rd edition (Prentice-Hall, NJ).

Morgül, Ö. \& Solak, E. [1996] "On the observer based synchronization of chaotic systems," Phys. Rev. E54, 4803-4811.

Morgül, Ö. \& Solak, E. [1997] "On the synchronization of chaotic systems by using state observers," Int. J. Bifurcation and Chaos 7, 1307-1322. 
Morgül, Ö. [1999] "Necessary condition for observerbased chaos synchronization," Phys. Rev. Lett. 82, 169-176.

Morgül, Ö. [2003] "A model-based scheme for anticontrol of some chaotic systems," Int. J. Bifurcation and Chaos 13, 3449-3457.

Schiff, S. J., Jerger, K., Duang, D. H., Chang, T., Spano, M. L. \& Ditto, W. L. [1994] "Controlling chaos in the brain," Nature 370, 615-620.

Solak, E., Morgül, Ö. \& Ersoy, U. [2001] "Observerbased control of a class of chaotic systems," Phys. Lett. A279, 47-55.

Ushio, T. [1999] "Synthesis of synchronized chaotic systems based on observers," Int. J. Bifurcation and Chaos 9, 541-546.

Wang, X. F. \& Chen, G. [2000a] "Chaotifying a stable LTI system by tiny feedback control," IEEE Trans. Circuits Syst.-I 47, 410-415.

Wang, X. F. \& Chen, G. [2000b] "Chaotification via arbitrarily small feedback controls: Theory, methods and applications," Int. J. Bifurcation and Chaos 10, 549-570.

Yang, W., Ding, M., Mandell, A. J. \& Ott, E. [1995] "Preserving chaos: Control strategies to preserve complex dynamics with potential relevance to biological disorders," Phys. Rev. E51, 102-110. 